\title{
The standardized exposure index for digital radiography: an opportunity for optimization of radiation dose to the pediatric population
}

\author{
J. Anthony Seibert $\cdot$ Richard L. Morin
}

Received: 9 July 2010 /Revised: 30 July 2010 / Accepted: 4 October 2010

(C) The Author(s) 2011. This article is published with open access at Springerlink.com

\begin{abstract}
The exposure index is currently a method by which digital radiography manufacturers provide feedback to the technologist regarding the estimated exposure on the detector, as a surrogate for image signal-to-noise ratio and an indirect indication of digital image quality. Unfortunately, there are as many exposure index values and methods as there are manufacturers, and in an environment with multiple vendors and a need to share data across institutions and dose registry databases, the situation is complicated. Fortunately, a new exposure index of digital X-ray imaging systems has been implemented. Developed concurrently by the International Electrotechnical Commission and the American Association of Physicists in Medicine in cooperation with digital radiography system manufacturers, the index has been implemented as an international standard. As explained, the exposure index does not indicate patient dose but rather a linearly proportional estimate of the incident radiation exposure to the detector. However, the use of the standardized exposure index and its associated target exposure index and deviation index values will likely lead to improved technologist performance in terms of uniformity and use of optimized radiographic techniques, leading to safer care of children needing radiographic examinations. Radiologists will benefit from standardized terminology, and institutions and clinics will be able to compare exposure index values with others through a
\end{abstract}

\footnotetext{
J. A. Seibert $(\triangle)$

Department of Radiology, University of California, Davis,

4860 Y St., Suite 3100,

Sacramento, CA 95817, USA

e-mail: jaseibert@ucdavis.edu

R. L. Morin

Department of Radiology, Mayo Clinic Jacksonville, Jacksonville, FL 32224, USA
}

national dose index registry database now under development. The Alliance for Radiation Safety in Pediatric Imaging, in its role as a benefactor of and advocate for the pediatric patient, is using the Image Gently campaign to disseminate information regarding the exposure index standard for digital radiography so that these benefits can be achieved in a rapid and effective manner.

Keywords Digital radiography - Digital radiography exposure index · Pediatric radiography radiation dose . Radiography exposure index standard . ACR dose index registry

\section{Introduction}

The exposure index (EI) in digital radiography has been used to indicate the relative speed and sensitivity of the digital receptor to incident X-rays and, ideally, to provide feedback to the technologist regarding the proper radiographic techniques for a specific exam that achieves an optimal image in terms of appropriate quality and corresponding low dose to the patient. Currently, because of the many proprietary and distinctly different ways of determining and reporting the EI by the manufacturers of digital radiography equipment, there has been confusion regarding use of this value from the technologist, radiologist and physicist communities. An effort to standardize the EI for all digital radiography detector systems was initiated by the International Electrotechnical Commission (IEC) and the American Association of Physicists in Medicine (AAPM) with participation by diagnostic physicists from around the world and representatives from many digital radiography manufacturers $[1,2]$. The goals of each group were recently completed, with the resultant IEC 
standard 62494-1 "exposure index of digital X-ray imaging systems," which provides a unified method to generate an exposure index value, requires users to input a target exposure index $\left(\mathrm{EI}_{\mathrm{T}}\right)$ value for each exam, and indicates a deviation index (DI) value that gives feedback to the technologist regarding technique and image quality based on signal-to-noise ratio [1]. Although these are important values in providing feedback in regard to achieving optimal images at the lowest dose, the EI and DI don't directly indicate patient dose. Dose to the patient is dependent upon many factors, including the parts of the body being examined, presence or lack of radiation-sensitive organs being exposed to X-rays, the area of the X-ray beam (determined by collimation) irradiating the patient, the output of the X-ray tube as a function of $\mathrm{kVp}$, tube current, exposure time and beam filtration. There are several ways to estimate the dose to the patient. Peak skin dose and effective dose are the most common. Peak skin dose values are determined from (1) measuring the X-ray tube output (air kerma per $\mathrm{mAs}$ as a function of $\mathrm{kVp}$ at fixed filtration) at a fixed distance and determining incident air kerma to the patient based upon knowledge of the $\mathrm{kVp}$ and $\mathrm{mAs}$ and corrections for variation in distance; (2) using a kerma area product (KAP) or reference dose device positioned at the Xray tube collimator that physically measures $\mathrm{x}$-radiation emanating from the focal spot, to generate a dose area product or a reference point dose, either of which can provide an estimate of the incident dose to the patient. Effective dose is a calculated value based upon the estimated dose to radiation-sensitive tissues and organs in the beam as the sum of the product of fractional estimated dose to organs/ tissues times their weighting coefficients.

Although the EI doesn't directly relate to patient dose, the importance of tracking and evaluating the EI and DI values in an effort to ensure correct use of the equipment and to optimize radiation dose on an exam-by-exam basis for appropriate patient care cannot be underestimated.

\section{What is radiation dose?}

Radiation dose is a measure of the amount of energy per unit mass absorbed in tissues from an X-ray beam, and is expressed in joules (unit of energy) per kilogram (unit of mass). The unit of radiation dose is the gray (Gy), equal to one joule/kilogram. To put this in perspective, the natural background radiation from radioactive materials in the earth and cosmic rays from space during the period of 1 year deliver about 0.003 Gy or $3 \mathrm{mGy}$. The skin dose delivered from a properly exposed chest radiograph to an averagesize man is about $0.15 \mathrm{mGy}$. Because there are different types of ionizing radiation, such as high energy protons, neutrons and heavier nuclei, the energy deposition and absorption can be concentrated in the cells and have a greater consequence in terms of biological effects. To normalize these biological effects, the unit sievert (Sv) is used for dose equivalent measures and effective dose. For radiographs, the dose of $1 \mathrm{~Gy}$ is equal to a dose equivalent of $1 \mathrm{~Sv}$. However, an entrance skin dose of $0.15 \mathrm{mGy}$ in the chest radiograph example delivers an effective dose of about $0.04 \mathrm{mSv}$. The peak skin dose is useful for evaluation of potential deterministic effects of ionizing radiation (e.g., radiation burn, hair loss and other acute effects) at very high radiation dose, while the effective dose estimate is useful for stochastic effects such as cancer induction probability. When expressing dose estimates to the patient, both measures are useful.

\section{How is patient radiation dose expressed for radiographic studies?}

There are many ways of expressing radiation dose to the patient from radiographic imaging studies. One is entrance skin dose, the maximum dose delivered by the incident radiation from the X-ray tube. Another measure is integral dose, which is determined by the characteristics of the patient, the area irradiated and amount of X-rays absorbed in the patient. A third is organ dose, which is dependent on the location of the procedure and the proximity of organs in the X-ray field and close by. The effective dose is an estimate of the dose to the whole body from a procedure that only irradiates a fraction of the total body, as with most radiographic studies. The effective dose is a value sometimes used to estimate probability of carcinogenic effects, for a population, based upon the sensitive organs that receive dose during the procedure. Specific organweighting factors and the radiation dose to the organs are multiplied and then summed to calculate effective dose, which is much less than the corresponding peak dose or integral dose.

\section{What level of patient dose should be allowed?}

Radiation dose delivered to a patient is a necessary consequence of acquiring the X-ray images used to define the anatomical and patho-physiological processes and make a diagnosis. Because X-rays are carcinogenic and have an associated risk, it is important to ensure that benefits of making an accurate diagnosis outweigh the risks of being exposed to ionizing radiation. Fortunately, the risks of being exposed to ionizing radiation in quantities typically used for medically indicated imaging procedures are quite low and similar to other risks that are deemed acceptable for everyday life. Thus, the 
radiographic study should be optimized in terms of achieving necessary image quality at the lowest possible radiation dose, in order to maximize the benefit-to-risk ratio. Note that this is not necessarily the lowest dose possible, but the minimal radiation dose that results in image quality sufficient to allow a competent radiologist to make a confident diagnosis. As long as the examination is appropriate, the benefit to an individual patient (to confirm or eliminate disease or trauma) will far outweigh the associated risk.

\section{Patient radiation dose and the exposure index for digital radiographic detectors: are they the same?}

No. The dose to the patient is determined by the X-ray technique factors $(\mathrm{kV}, \mathrm{mAs}$, grid, SID, filtration, beam collimation), X-ray beam penetrability and quality, the amount of energy imparted to the body, and the size and area of the body irradiated. The exposure incident on the detector is determined by the remnant radiation (primary radiation transmitted through the patient and scattered radiation transmitted from the patient) that is absorbed, converted to electronic signals, and formatted into a digital radiographic image with a given detective quantum efficiency (DQE). The DQE is a measure of information transfer efficiency that is dependent not only on the efficient absorption of X-rays but also on the conversion into a useful signal with minimum corruption by other detector noise sources (such as electronic and artifact noise). The exposure index is a measure of the signal level produced by a digital detector for a given incident exposure transmitted through the patient, is proportional to the signal-to-noise ratio squared $\left(\mathrm{SNR}^{2}\right)$, and is related to image quality.

So, if a large patient is imaged on a digital detector through a thick body part, the X-ray technique must be adjusted upward to compensate for X-ray attenuation and to ensure a sufficient number of transmitted X-rays incident on the detector in order to achieve a desired exposure index value. This represents a relatively high dose to the patient. Now if a thin patient is imaged on the same detector, the Xray technique must be adjusted downward to achieve the same number of transmitted X-rays to the detector (for the same $\mathrm{SNR}^{2}$ ), with the result being the same exposure index. Thus, without knowledge of the X-ray acquisition techniques and patient habitus, it is not possible to directly calculate the patient dose from the exposure index value. However, since digital radiography devices have the ability to correct for under- and overexposure conditions, the exposure index can give the technologist an idea of whether a proper radiographic technique was used. This is extremely important in the effort to optimize radiographic studies and acquisition techniques, especially for children, in whom radiation sensitivity is relatively high.

\section{Exposure index for digital radiography detectors}

Radiography has played an important role in the diagnosis and management of patients for more than 110 years. Traditional screen-film systems use overall film density as an exposure indicator. Direct feedback to the technologist regarding exposure is obtained by the appearance of the processed film image (Fig. 1). Optimized technique factors $(\mathrm{kVp}$ and $\mathrm{mAs})$ are based upon the patient size and body part and radiographic speed of the screen film combination being used. Particularly in situations where automatic exposure control is not used (for instance, in the majority of small pediatric patients), the use of fixed speed screenfilm detectors requires the technologist to use experience and appropriate judgment to set radiographic techniques.

Since the mid-1990s, a steady replacement of analog screen-film detectors with digital radiography (DR) detectors has occurred, along with an expectation of lower dose because of minimal retakes and consistent image quality. This is because computed radiography (CR) and direct radiography devices (herein collectively referred to as digital radiography - DR) have wide exposure latitude, a variable speed class of operation, and image postprocessing capabilities that provide consistent image appearance even with underexposed and overexposed radiographs (Fig. 2). Determining correct radiographic techniques and patient exposure by image appearance (e.g., density on a film image) is no longer possible. While underexposed images have smaller numbers of X-rays absorbed by the digital detector and can be recognized by a noisy appearance (Fig. 2), overexposed images can easily go unnoticed, resulting in needless overexposure and potential harm to the patient.

Digital radiography systems use image processing to align the histogram values with the look-up-table translation curve, such that the image gray-scale is similar over a wide range of image exposures. As shown, direct visual cues (dark and light) are lost regarding exposure. While the image processing adjusts the gray-scale, images with low signals will have increased noise and mottle, whereas images with high signal are associated with good image quality but high patient dose (Fig. 3). An underexposed image has high noise content, and therefore can be readily recognized. However, an overexposed image has low noise and appears to be very acceptable, but without some indication of incident exposure level to the detector, this overexposure can go unrecognized with the corresponding needless extra radiation dose to the patient. In fact, overexposures 5-10 times a normal exposure will have 

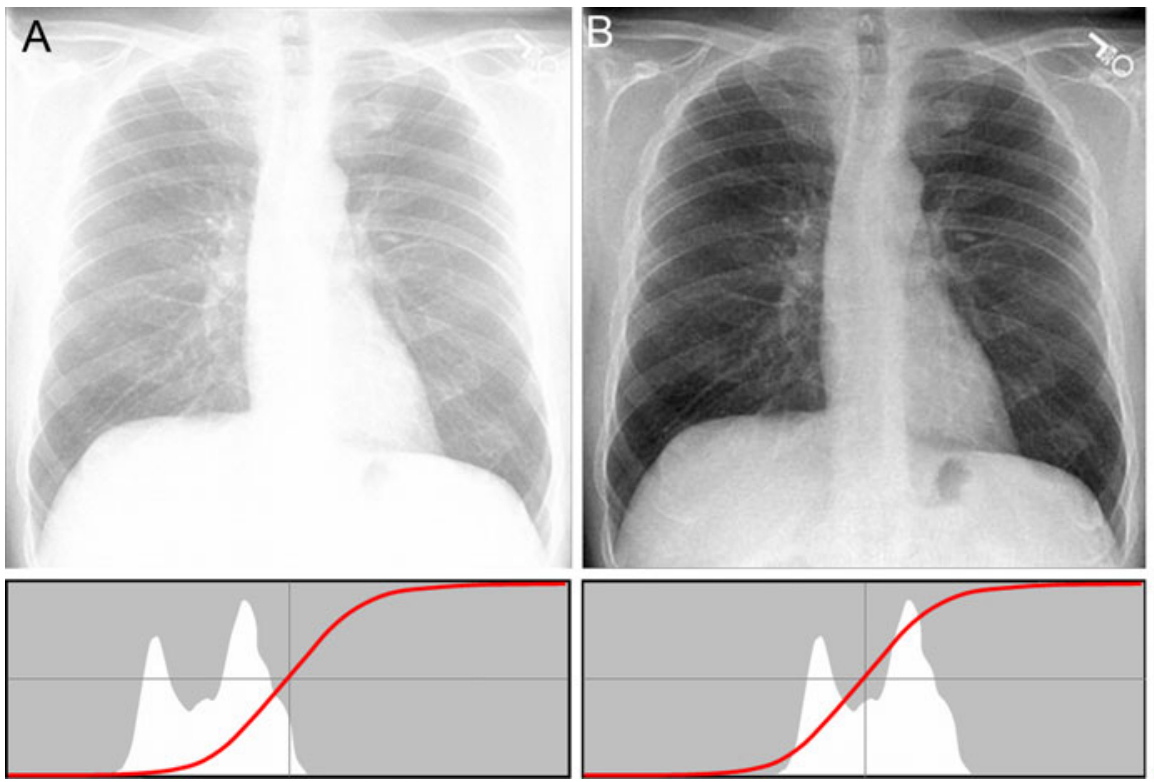
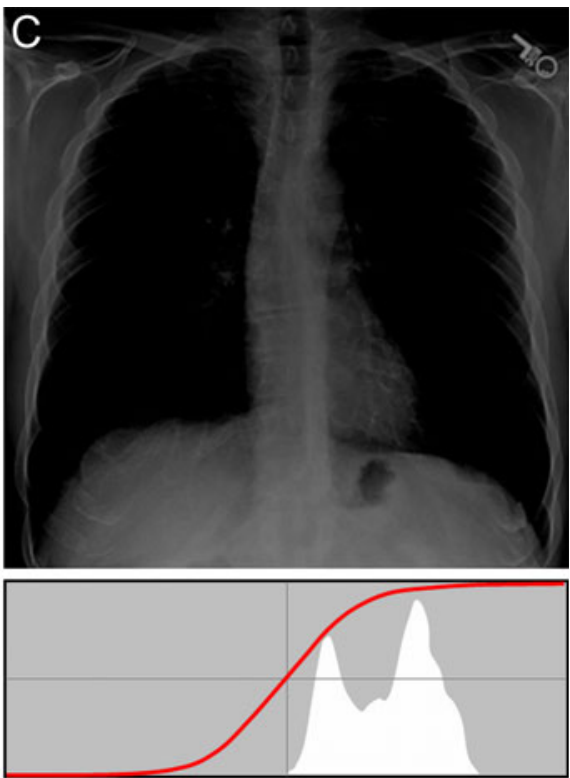

Fig. 1 Shown are the responses of a screen-film detector with a fixed radiographic speed to (a) underexposure, (b) correct exposure and (c) overexposure. Underneath each image is a histogram representing the intensity of X-rays transmitted through the patient. The $\mathrm{x}$-axis represents transmitted exposure intensity and the y-axis represents magnitude. The s-shape line is the characteristic curve that translates exposure intensity into optical density on the processed film. Note that this curve does not change position along the exposure axis the appearance of a properly exposed image, because of compensation by the digital detector. Lack of a feedback indicator and a lack of understanding of what the exposure indicator means can lead to needless patient dose, or "dose creep" [3].
Digital radiography system manufacturers have recognized the need for methods to indicate incident exposure index levels and have provided many proprietary ways to describe the feedback signals (or in some cases, no feedback at all). Unfortunately, widely different methods
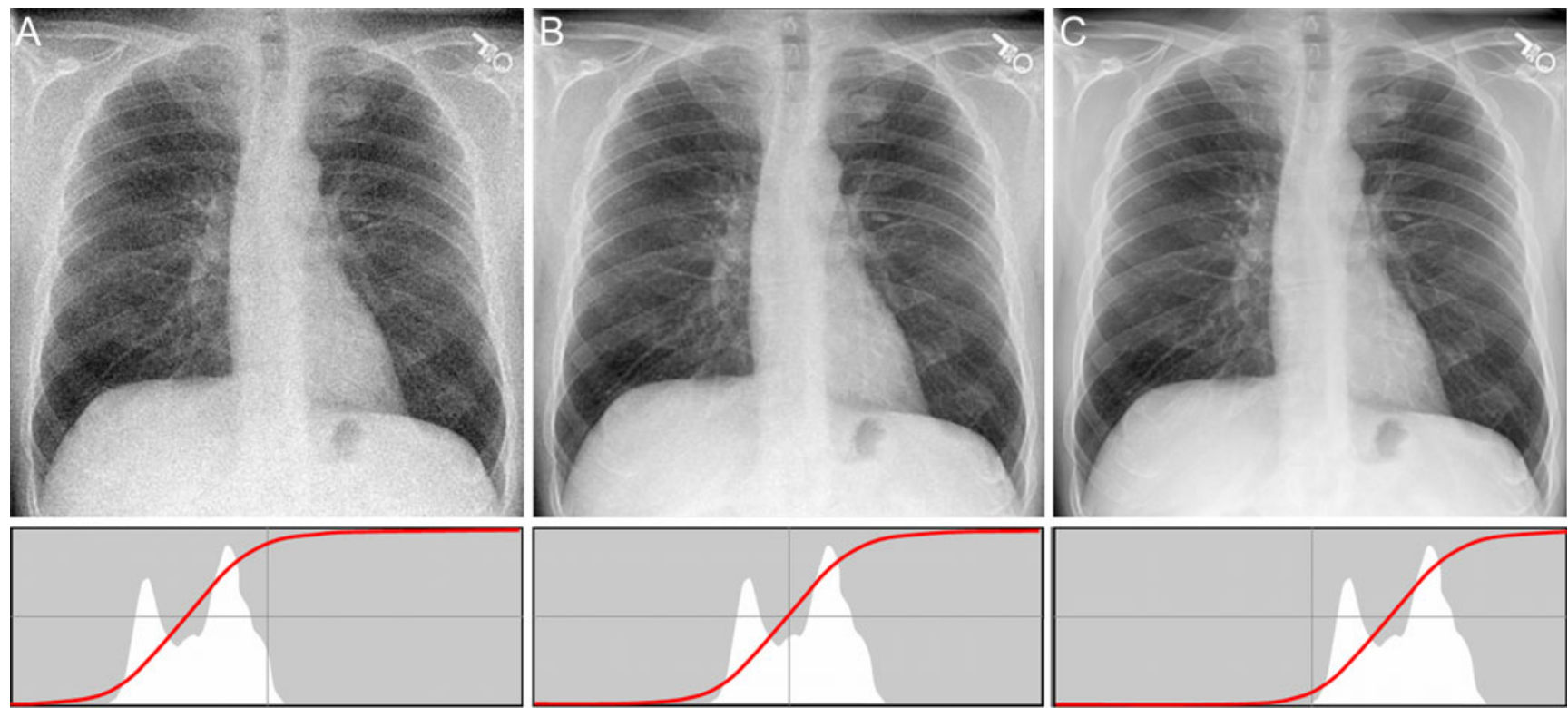

Fig. 2 Response of a digital detector to exposure intensity variations. a Underexposure. b Correct exposure. c Overexposure. Underneath each image is a histogram representing the frequency distribution of digital values (directly related to intensity of X-rays transmitted through the patient, as in Fig. 1). The $\mathrm{x}$-axis represents digital value and the $y$-axis represents frequency. The s-shape line is the characteristic curve (a digital value of interest look-up-table, VOILUT) that translates raw digital value into a contrast- and brightnessoptimized image ready for presentation. The VOILUT is adjusted to the histogram to achieve optimal rendering of the image content 


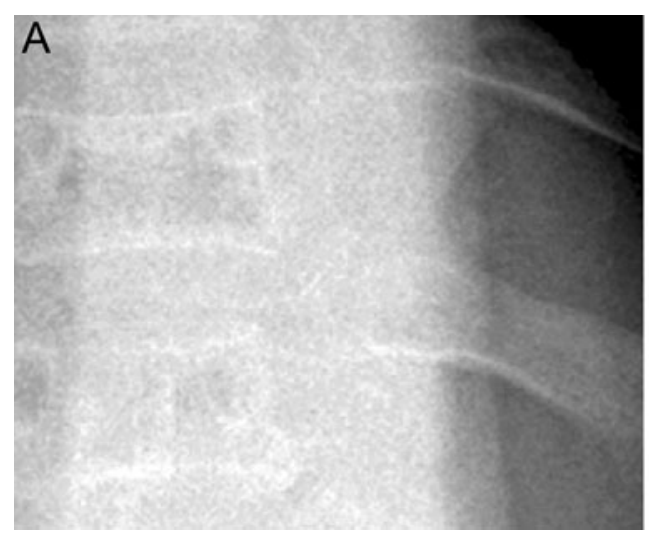

Fig. 3 Underexposed vs. overexposed images. a Example of an underexposed image illustrates the quantum mottle caused by a low number of X-ray photons to the detector, and corresponding low dose to the patient. However, depending on the diagnostic need, is there adequate image quality to make the diagnosis? b Example of an

to calculate the exposure index value have evolved, as shown in Table 1. The variability in the reported exposure index values has led to widespread confusion and frustration in the imaging community.

Digital radiography image processing algorithms assess the recorded raw data ("for processing") signals to indicate whether the signal-to-noise ratio (SNR) is in the appropriate range, and thus indirectly whether the radiographic technique is appropriate for the patient being imaged. Tests with manufacturer-defined beam conditions are used to verify that correct indicators are being reported. Recommended exposure indicator ranges are used by technologists to check each radiographic exposure. Digital detector systems have exposure indicators that estimate the amount of radiation to the detector based on several methods: (1) the analysis of a segmented region or regions within the whole image; (2) regular region locations in the image; (3) specific anatomical regions in the image that depend on the examination type selected. Each type of analysis uses the recorded information, typically in a histogram form but sometimes as a summed value, to identify the average signal strength within the region by comparing its histogram shape to a known shape of a similar image.

Using the values as expressed in Table 1, the approximate EI value versus incident receptor exposure is shown in Table 2. The widely different EI values for equivalent receptor exposure represent clear evidence of the need for an exposure indicator standard.

\section{Standardized exposure index for digital radiography: technical issues}

The American Association of Physicists in Medicine (AAPM), in cooperation with many manufacturer repre-

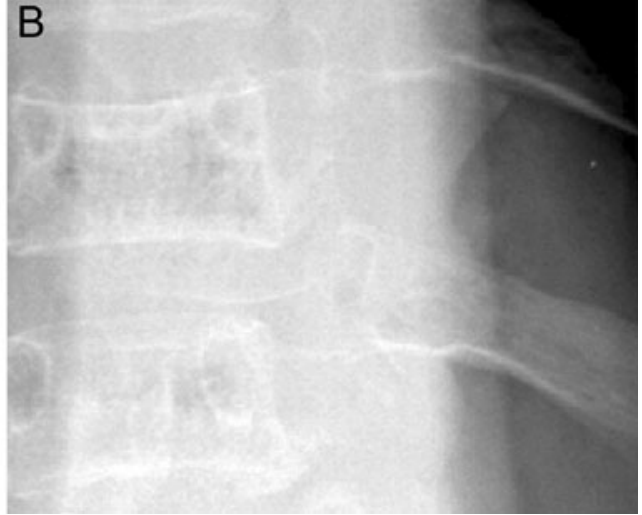

overexposed image illustrates excellent contrast sensitivity and detail but also a much higher dose to the patient. In this case, while minimal quantum mottle and excellent image quality is achieved, is the patient overexposed and, if so, by how much?

sentatives and the Medical Imaging and Technology Alliance (MITA), and in collaboration with the International Electrotechnical Commission (IEC), has developed a methodology that has evolved into an international standard, IEC 62494-1 [1], that describes common exposure indices and deviation indices to be implemented across all digital radiography detector types and across all manufacturers and vendors of such equipment. The document explains a method for placing standardized exposure information and content in the DICOM metadata in each image associated with the imaging study. While the details are left to the interested reader [1,2], it is the manufacturer's responsibility to calibrate the imaging detector according to a detector-specific procedure, to provide methods to segment pertinent anatomical information in the relevant image region and to generate an exposure index (EI) that is linearly proportional to detector exposure.

The user's responsibility is to define each patient and anatomy-specific examination with a target exposure index $\left(\mathrm{EI}_{\mathrm{T}}\right)$ for the specific detector in use. Determination of $\mathrm{EI}_{\mathrm{T}}$ values is crucial for the successful implementation of the exposure index standard and requires assessment of image quality. This is an often subjective endeavor, causing a wide variation in response with respect to recommended $k V p$ as well as exposure to the detector from the many stakeholders involved in the process. A significant ongoing effort by the Alliance for Radiation Safety in Pediatric Imaging through the Image Gently campaign has pushed for scientific methods that can objectively establish target values for optimizing the quality of the image while minimizing radiation dose and improving overall care and safety for pediatric (and all) patients.

After image acquisition and any manual adjustments of the automatic processing that updates the EI (e.g., if the 
Table 1 Manufacturer and exposure index parameters used for digital radiography systems

\begin{tabular}{|c|c|c|c|c|c|}
\hline Manufacturer & Exposure indicator name & Symbol & Units & Exposure dependence, $\mathrm{X}$ & Detector calibration conditions \\
\hline Fujifilm & $\mathrm{S}$ value & S & Unitless & $200 / \mathrm{S} \propto \mathrm{X}(\mathrm{mR})$ & $\begin{array}{l}80 \mathrm{kVp}, 3 \mathrm{~mm} \\
\text { Al "total filtration" } \\
S=200 @ 1 \mathrm{mR}\end{array}$ \\
\hline Carestream & Exposure index & EI & Mbels & $E I+300=2 X$ & $\begin{array}{l}80 \mathrm{kVp}, 1.0 \mathrm{~mm} \\
\mathrm{Al}+0.5 \mathrm{~mm} \\
\mathrm{Cu} ; \mathrm{EI}=2000 @ 1 \mathrm{mR}\end{array}$ \\
\hline Agfa & $\begin{array}{l}\text { Log of median } \\
\text { of histogram }\end{array}$ & $\lg \mathrm{M}$ & Bels & $\lg M+0.3=2 \mathrm{X}$ & $\begin{array}{l}400 \text { speed class, } \\
75 \mathrm{kVp}+1.5 \mathrm{~mm} \\
\mathrm{Cu} ; 1 \mathrm{gM}=1.96 @ 2.5 \mu \mathrm{Gy}\end{array}$ \\
\hline Konica & Sensitivity number & $\mathrm{S}$ & Unitless & For $\mathrm{QR}=\mathrm{k}, 200 / \mathrm{S} \propto \mathrm{X}(\mathrm{mR})$ & $\begin{aligned} \mathrm{QR} & =200,80 \mathrm{kVp} \\
\mathrm{S} & =200 @ 1 \mathrm{mR}\end{aligned}$ \\
\hline Canon & Reached exposure value & REX & Unitless & $\begin{array}{l}\text { Brightness }=c_{1}, \text { Contrast }=c_{2}, \\
\text { REX } \propto X^{1}\end{array}$ & $\begin{array}{l}\text { Brightness }=16 \\
\text { Contrast }=10 \\
\mathrm{REX} \approx 106 @ 1 \mathrm{mR}^{1}\end{array}$ \\
\hline Canon & EXP & EXP & Unitless & $\mathrm{EXP} \propto \mathrm{X}$ & $\begin{array}{l}80 \mathrm{kVp}, 26 \mathrm{~mm} \\
\mathrm{Al}, \mathrm{HVL}=8.2 \mathrm{~mm} \\
\mathrm{Al}, \mathrm{DFEI}=1.5 \\
\mathrm{EXP}=2000 @ 1 \mathrm{mR}\end{array}$ \\
\hline GE & $\begin{array}{l}\text { Uncompensated } \\
\text { detector exposure }\end{array}$ & UDExp & $\mu \mathrm{Gy}$ air kerma & UDExp $\propto X(\mu G y)$ & $\begin{array}{l}80 \mathrm{kVp} \text {, standard } \\
\text { filtration, no grid }\end{array}$ \\
\hline GE & $\begin{array}{l}\text { Compensated } \\
\text { detector exposure }\end{array}$ & CDExp & $\mu \mathrm{Gy}$ air kerma & CDExp $\propto X(\mu \mathrm{Gy})$ & Not available \\
\hline GE & Detector exposure index & DEI & Unitless & $\begin{array}{l}\text { DEI } \approx \text { ratio of actual } \\
\text { exposure to expected } \\
\text { exposure scaled by technique } \\
\text { and system parameters. } \\
\text { Expected exposure values } \\
\text { can be edited by user as } \\
\text { preferences. }\end{array}$ & Not available \\
\hline Swissray & Dose indicator & DI & Unitless & Not available & Not available \\
\hline Imaging Dynamics & Accutech & F\# & Unitless & $2^{\mathrm{fH}}=\mathrm{X}(\mathrm{mR}) / \mathrm{X}_{\mathrm{tgt}}(\mathrm{mR})$ & $80 \mathrm{kVp}+1 \mathrm{~mm} \mathrm{Cu}$ \\
\hline Philips & Exposure index & EI & Unitless & 1000/X ( $\mu \mathrm{Gy})$ & $\begin{array}{l}\text { RQA5, } 70 \mathrm{kV},+21 \mathrm{~mm} \\
\mathrm{Al}, \mathrm{HVL}=7.1 \mathrm{~mm} \mathrm{Al}\end{array}$ \\
\hline Siemens & Exposure index & EXI & $\mu \mathrm{Gy}$ air kerma & $\mathrm{X}(\mu \mathrm{Gy})=\mathrm{EI} / 100$ & $\begin{array}{l}\text { RQA5, } 70 \mathrm{kV}+0.6 \mathrm{~mm} \\
\mathrm{Cu}, \mathrm{HVL}=6.8 \mathrm{~mm} \mathrm{Al}\end{array}$ \\
\hline Alara CR & Exposure indicator value & EIV & Mbels & $\mathrm{EIV}+300=2 \mathrm{X}$ & $\begin{array}{l}1 \mathrm{mR} \text { at RQA5, } \\
70 \mathrm{kV},+21 \mathrm{~mm} \\
\mathrm{Al}, \mathrm{HVL}=7.1 \mathrm{~mm} \\
\mathrm{Al}=>\mathrm{EIV}=2000\end{array}$ \\
\hline iCRco & Exposure index & None & Unitless & $\begin{array}{l}\text { Exposure index } \propto \\
\log [\mathrm{X}(\mathrm{mR})]\end{array}$ & $\begin{array}{l}1 \mathrm{mR} @ 80 \mathrm{kVp}+1.5 \mathrm{~mm} \\
\mathrm{Cu}=>0\end{array}$ \\
\hline
\end{tabular}

Table 2 Manufacturer, corresponding symbol for EI and calculated EI for three incident exposures to the detector. Note: these are approximate relationships because of different calibration conditions for the various manufacturers

\begin{tabular}{lllll}
\hline Manufacturer & Symbol & $5 \mu \mathrm{Gy}$ & $10 \mu \mathrm{Gy}$ & $20 \mu \mathrm{Gy}$ \\
\hline Canon (brightness $=16$, contrast $=10)$ & REX & 50 & 100 & 200 \\
IDC (ST=200) & F\# & -1 & 0 & 1 \\
Philips & EI & 200 & 100 & 50 \\
Fuji, Konica & S & 400 & 200 & 100 \\
Carestream (CR, STD) & EI & 1,700 & 2,000 & 2,300 \\
Siemens & EI & 500 & 1,000 & 2,000 \\
\hline
\end{tabular}


anatomical segmentation fails to recognize relevant image regions, such as the presence of a prosthesis, a gonadal or breast shield commonly used in pediatric radiography, or other unlikely attenuator causing the displayed image to appear suboptimal), a feedback signal known as the deviation index (DI) is calculated according to:

$\mathrm{DI}=10 \log _{10}\left(\mathrm{EI} / \mathrm{EI}_{\mathrm{T}}\right)$

The DI provides feedback to the operator with a value that is equal to 0 when the appropriate exposure to the detector is achieved, a negative number when underexposure has occurred, and a positive number when overexposure has occurred. A DI value of +1 indicates an overexposure equal to $25 \%$ more than the target exposure to the detector, while a value of -1 indicates an underexposure equal to $20 \%$ less than desired. The range of DI values acceptable for routine clinical work needs further investigation. The range will probably be narrower for examinations using automatic exposure control versus manual parameter setting. DI values of +3 and -3 indicate exposures that are 2 times more and less than the target exposure, respectively.

The DI is intended to be an indication to the technologist on whether the radiographic technique is appropriate for the specific body part and view for an optimal image presentation of the anatomy of interest (proper image brightness and contrast) with acceptable signal-to-noise ratio in the relevant image regions. Therefore, a database of $\mathrm{EI}_{\mathrm{T}}$ values must be available in the digital X-ray imaging system for each imaging procedure, and the technologist must specify the correct body part and radiographic view. Otherwise, the calculated DI might be inaccurate because an incorrect $\mathrm{EI}_{\mathrm{T}}$ value could be used. In imaging situations where deviations from proper positioning and collimation occur, resulting in under- or overexposure in screen-film imaging (for instance, where there is improper positioning of anatomy over an AEC chamber or too small or too large of a collimation area, resulting in lesser or greater amounts of scatter onto the detector and causing the optical density of the processed film to be under- or overexposed), the proper use of the exposure index standard will result in negative or positive values of the DI.

There are many limitations in the use of the standardized EI. The generated EI value depends critically on the relevant image region analyzed; as a result, different EI values are possible when a different image region is segmented and used for estimating the incident exposure. Manufacturers use different analysis methods to determine the relevant image regions and corresponding image histograms. X-ray detectors can have widely different detection efficiencies and respond differently to X-rays of different energies and angles of incidence. Also, the EI is calibrated to only one acquisition condition ( $\mathrm{kV}$, filtration, SID, grid) and to the extent that the X-ray energies incident on the detector from a radiographic acquisition are different, inaccuracies in the calculated value can result. Even though the same EI value is reported by different systems, the exposure reaching the detector might be very different. Likewise, significantly different EI values do not necessarily indicate significantly different exposure to the digital X-ray detector. Nevertheless, the ability to overcome segmentation errors to allow for re-computation of the EI by manual adjustment of the image display characteristics and to provide consistent operator feedback via the calculated DI will undoubtedly reduce the confusion and enhance the understanding and safe use of digital radiography detector systems, in particular for pediatric technique factors. The nuances of this new exposure index standard now are at the beginning of clinical implementation and testing, so the next steps are to figure out the appropriate $\mathrm{EI}_{\mathrm{T}}$ for specific detectors and examinations, to understand the limitations of the EI and to quickly learn about the DI and how to properly compensate for under- and over-exposures with the DI information presented to the technologist as feedback.

\section{The American College of Radiology dose index registry}

The American College of Radiology (ACR) dose index registry (DIR) was established to provide a mechanism to automatically extract dose index information from image headers and forward them to a central source to compile a registry of dose indices for specific procedures. With this program, a facility or an individual could periodically interrogate the registry to get their dose indices for procedures and compare their values against local, regional, national and international results. Hence, this tool could be used in an ongoing basis by either a quality committee or a radiation safety committee to periodically assess the relative amounts of radiation used for specific imaging procedures. The concept is to use the optimal amount of radiation to provide a diagnostic image. If the index is too low, that might indicate that image quality is suffering. If the dose index is too high, that might imply use of more radiation than necessary for diagnostic imaging. Schematics of the process are given in Fig. 4.

The images are sent from the modality to an independent workstation usually for quality control, and then on to the servers for image interpretation. The images can also be sent from either the modality or quality control to an independent computer, upon which resides the ACR software to extract DICOM data elements (including the dose indices CTDI $_{\mathrm{vol}}$ and DLP) from the DICOM header. These records are then uploaded to the ACR DIR, either in 
Fig. 4 The process of data acquisition and collection, extraction of dose information and delivery to the dose index registry database is shown
ACR Dose Index Registry

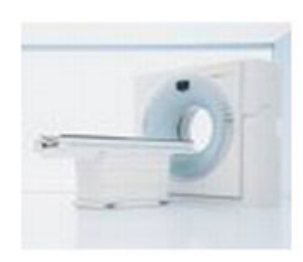

a transactional manner or for a single transmission at a specific time. The first pilot for the ACR DIR was using dose indices from computed radiography systems. These initial records determined that the concept was valid and that the registry could be built. One distinctive problem was the variation among dose indices (Table 1). With the standardization of the exposure index, the ACR DIR Committee is prepared to return to entry of digital radiography data into the registry in 2011. This will enhance the ability of facilities (particularly in pediatrics) to continuously monitor the levels of radiation used in imaging their patients. After the CR/DR pilot experience, the DIR turned its attention to CT. The CT DIR pilot demonstrated interesting and challenging results, one of which was the advice of an ancient Chinese proverb: "The beginning of wisdom is to call things by their right names." It was discovered that the nonstandard naming conventions for exams contributed to a rather substantial problem. One particular exam of the pelvis was described by more than 40 names. Participants in the next CT DIR trial will have the opportunity to submit their charge master and their charge master entries will be harmonized with the RadLex Lexicon, hence standardizing the naming of CT examinations. The ACR DIR has great potential to assist in the goals of the Image Gently campaign with regard to digital radiography radiation exposures. In addition, this technique could be a useful measure in physician performance improvement or ABR Maintenance of Certification PQI Projects. All of these efforts will assist in driving the community toward the use of optimal radiation for highquality imaging of pediatric patients.

\section{What are the responsibilities of the user in this new "exposure index standard" paradigm?}

1. When purchasing new digital and computed radiography equipment, insist on the implementation of the EI standard, IEC 62494-1.

2. For older direct radiography and computed radiography equipment, request from the manufacturer software that complies with the new EI standard. (Note that the likelihood of this capability diminishes with the age of the equipment.)

3. Understand and get training on the manufacturer's software that implements the EI standard. Prepare a list of $\mathrm{EI}_{\mathrm{T}}$ values for all radiographic procedures for input into the digital detector database, and include these in acquisition protocols. Continuous updating, as experience allows, results in enhanced optimization and increased benefit-to-risk for the patients having a radiographic procedure.

4. Record EI, $\mathrm{EI}_{\mathrm{T}}$, DI and performing technologist for all studies, and review values on a periodic basis; investigate the cause of frequent outliers. Train technologists on the use of the DI, acceptable limits of the DI value, and compensation methods when the DI is beyond the acceptable range.

5. Record the corresponding examination $\mathrm{kVp}$, mAs, filtration and acquisition geometry within the DICOM header (this might not be possible for passive computed radiography detectors or legacy or add-on digital equipment). These are the data needed to estimate patient radiation dose. 
6. Share experiences and data in order to provide the imaging community with baseline reference data from which practice improvements can be made for all users. The Alliance for Radiation Safety in Pediatric Imaging is playing a key role in providing an international presence that can assist in the distribution of pertinent information, so check in frequently to the website for updated information.

7. Participate in the ACR dose index registry.

Acknowledgement This article is based on presentations given by the authors at the Image Gently Digital Radiography Summit, St. Louis, MO, USA, on Feb. 4, 2010.

Open Access This article is distributed under the terms of the Creative Commons Attribution Noncommercial License which per- mits any noncommercial use, distribution, and reproduction in any medium, provided the original author(s) and source are credited.

\section{References}

1. International Standard IEC 62494-1 (2008) Medical electrical equipment - exposure index of digital X-ray imaging systemsPart 1: definitions and requirements for general radiography. International Electrotechnical Commission, ISBN 2-8318-99443

2. Shepard SJ, Wang J, Flynn M et al (2009) An exposure indicator for digital radiography: AAPM Task Group 116 (executive summary). Med Phys 36:2898-2914

3. Practice guideline for digital radiography (2007) American College of Radiology. Available via http:/www.acr.org/SecondaryMainMenu Categories/quality safety/guidelines/dx/digital radiography.asp. Accessed 17 June 2010 